CORRECTION

https://doi.org/10.1038/s41586-019-1412-z

\title{
Author Correction: Resonantly hybridized excitons in moiré superlattices in van der Waals heterostructures
}

Evgeny M. Alexeev, David A. Ruiz-Tijerina, Mark Danovich, Matthew J. Hamer, Daniel J. Terry, Pramoda K. Nayak, Seongjoon Ahn, Sangyeon Pak, Juwon Lee, Jung Inn Sohn, Maciej R. Molas, Maciej Koperski, Kenji Watanabe, Takashi Taniguchi, Kostya S. Novoselov, Roman V. Gorbachev, Hyeon Suk Shin, Vladimir I. Fal'ko \& Alexander I. Tartakovskii

Correction to: Nature https://doi.org/10.1038/s41586-019-0986-9, published online 6 March 2019.

In the Acknowledgements section of this Letter, the first grant number has been corrected from ' 696656 ' to '785219' in the sentence 'We acknowledge financial support from the European Graphene Flagship Core 2 project under grant agreement $785219 . .$. . The original Letter has been corrected online. 